\title{
A Google Map Based Social Network (GMBSN) for Exploring Information about a Specific Territory
}

\author{
Wasim Ahmad*, Ashraf Zia, Usman Khalid \\ Department of Computer Sciences, Abdul Wali Khan University Mardan, Shankar Campus, Khyber, Pakistan. \\ Email: *was_last@yahoo.com, ashrafzia@awkum.edu.pk, uknewfashion@gmail.com
}

Received April 30 $0^{\text {th }}, 2013$; revised June $1^{\text {st }}, 2013$; accepted June $10^{\text {th }}, 2013$

Copyright (C) 2013 Wasim Ahmad et al. This is an open access article distributed under the Creative Commons Attribution License, which permits unrestricted use, distribution, and reproduction in any medium, provided the original work is properly cited.

\begin{abstract}
Social Networking is a harbinger to a more recent era in the area of computing where allocated and central resources are used in an exclusive manner. Millions of people around the globe with access to the internet are part of one or more social networks. They have permanent online accounts on Facebook and Twitter etc. where they create profiles, share photos, videos, useful links, their thoughts and spend hours catching up with what their friends are doing in their lives. The problem arise when somebody needs specific information about any city inside a country e.g. Where he/she can live? What he/she can eat? Where is the best place for outing? What are the special events relevant to that region? And may be any other help? In this paper we suggest a social network called Google map based social network (GMBSN), where users can choose their desired city of interest from the list. The selected city will be highlighted on Google map. After choosing any city from the map, the user will be able to select any category from the list and start finding and sharing information about the desired city of any country.
\end{abstract}

Keywords: Google Map; Social Network; GMBSN; LBSN's; LARE; LDA

\section{Introduction}

The social networking sites like Facebook and Twitter have rapidly gained popularity in the recent decade. Millions of people have their online Facebook and Twitter accounts and connect to the worldwide web. Users are connected with each other by sharing their thoughts, pictures and videos etc. [1-3].

\subsection{Google Map}

The Google map is a mapping application provided by Google. One can embed it to one's site by highlighting their areas of business, office, restaurant, hospital etc. for easy access. Google map is a global map where users can find any area on the globe, Whereas Google map location can be created by user according to their need [4]. Google map is simple to customize, one can include zooming, dragging and other options as well easily. Google map only requires user's registration to register secret key of Google map API [5].

\subsection{Google Map API}

Google map has a large scale of APIs which enable the

\footnotetext{
"Corresponding author.
}

clients to embed their applications easily [6]. It can be used in desktop applications, web applications and mobile applications. In the midst of APIs congestion Google Maps JavaScript API v 3.9 is used that let one to penetrate one's dynamic page into web page and make utilities available to their customized map [7]. We can access Google map API as shown in Figure 1.

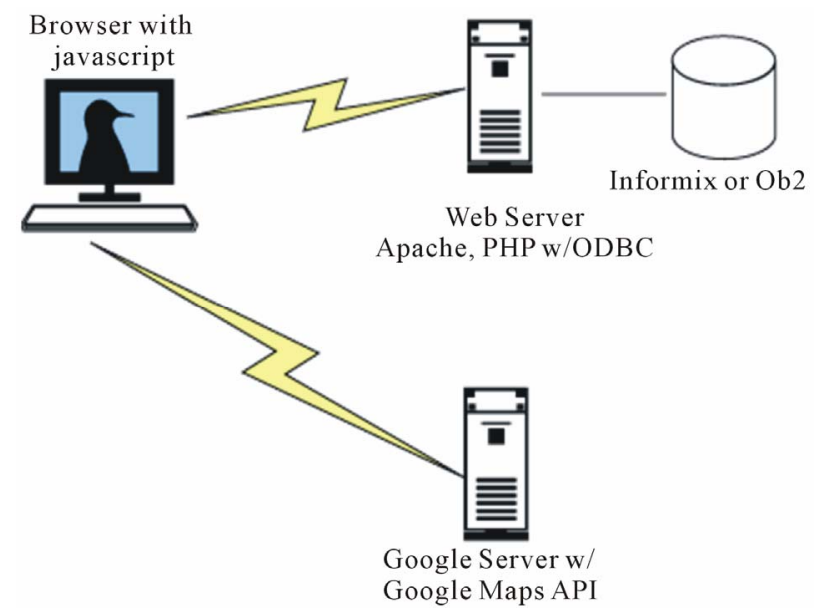

Figure1. Accessing google map API [8]. 


\subsection{Features}

- Any user can login to the GMBSN system by providing their Facebook account information for easy access.

- Since GMBSN supports not only PC but also mobile phones, users can access it from anywhere on the globe.

- Users can search/access any required information regarding any city of the target country from everywhere across the world 24/7.

By merging these features we have managed to develop such a system that gives the opportunity for every user to access and use it as a social network.

\section{User Authentication}

User authentication is required for rating a post, sharing new posts, photos, videos, links and allowing users to access GMBSN system. User will be required to login the network through Facebook account. Facebook API is used for the authentication due to its enormous data and users across the globe.

Facebook API (Application Programming Interface) is provided by Facebook for developers in order to access and submit information by programming [9]. Facebook API can be accessed by using the following Figure 2 .

\section{Literature Review}

Some map based SNS (Social Networking Sites) have already been developed. We can take example of shakabanga which provides Google map with list of the certain major countries. By clicking on any desirous country, users are then provided with some interesting images of the target country. Foursquare is another example, where users select their locations and can find some appropriate information related to that region.

Jia-Ching et al. [11] worked on user association analysis of locales on location based social networks. It has a solid abstract with the proposal of four locale based metrics. These metrics include locale clustering coefficient, Inward locale transitivity, locale assertively coefficient,

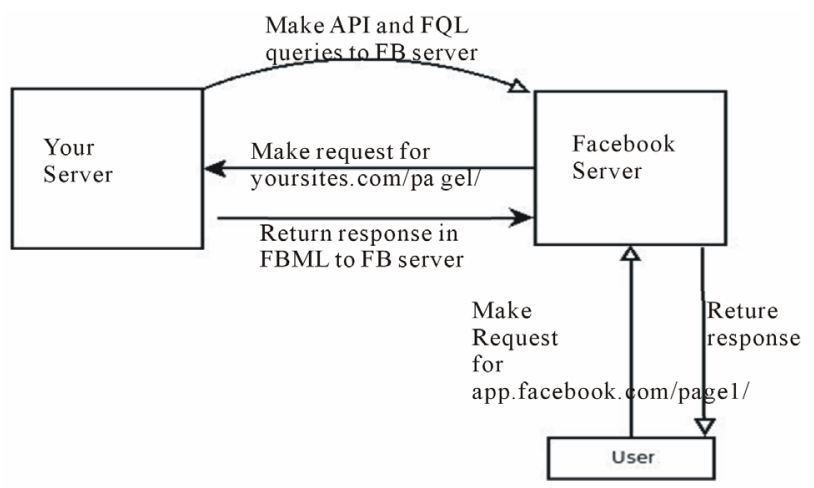

Figure2. Accessing Facebook API [10]. and locale assort ability coefficient. It has also the observation ability, when people who share more trajectories would get more attention and users who connect to other users who are already been popular. As for the future work, it has a plan to further investigate other locale based metrics to enhance the analysis on LBSNs. In FCF observation, social friends share more common locations than non-friends. According to the patio social analysis of foursquare observations nearby friends can share more common visited locations. It contains the comparison graphs of different approaches proposed in this paper. It has some ambiguous algorithms in some sections e.g. Inward locale transitivity and locale assertively coefficient. It also contains some ambiguous titles of the proposed metrics and does not contain any future enhancements for the proposed approaches.

Bao et al. [12] worked on location-based and preference-aware recommender system. This system provide the users with location recommendations around the specified geo-Position based on the user's personal preferences Learnt from their location history and social opinions piled up by the local experts who could share analogous interests. It also recommends a system which facilitates people to not travel only near their residential areas but also to a city that is new to them. It also proposes a novel location recommended system for people who travel to new cities. The main part of the proposed system is online recommendation and offline modeling. This paper also provides well understandable diagrams. There is conclusion in its algorithms and it also contains some ambiguous titles which need to be clarified. It does not have any progress of future enhancements.

Yamamoto et al. [13] worked on social networking service based on the location of students. It proposed a system that has the cited below functions.

1) Can obtain information regarding when and where the students are located by using both the attendance records of classes, and the login records of educational computers. These records are automatically recorded by a student card serving on a noncontact-type IC.

2) While system supports also web access form cell phone therefore users can also access it from outdoors. These functions enable users to search out anything anywhere in the vicinity without special terminals. One can expect the communication through this system not only on the web, but can too expect in real life. It has some well understandable diagrams that can be easily understand. This paper has absolutely fair functions, so that there remains no negative point in this paper.

Chuan Tan et al. [14] worked on Location aware applications for smart cities with Google maps and GIS tools. It presents the results of data analysis over the user profile, update activities, mobility characteristics, social graphs, and attribute correlations. It is the first large- 
scale quantitative analysis of a real-world commercial LSN service. This paper also states that that one user traveled 95,155 miles in 62 days, which means that the user traveled 1500 miles per day on average. It has a poor abstract which is difficult to understand. There are lots of complexities and has no future progress.

Scellato et al. [15] worked on Measuring User Activity on an Online Location-based Social Network. This paper presents measurement study of user activity on a popular online location-based social network with hundreds of thousands of users. It also describes that how the number of friends appears distributed according to a DoublePareto law, both the number of check-ins and the number of places per user are better described by normal distributions. Moreover it reports how user activity spans decay faster than exponentially and how, over time, users add friends more quickly than they accumulate check-ins and places. It also suggest that the difference in the distribution of friends and check-ins/places may be motivated by physical constraints that do not allow users to steadily visit very large numbers of new places, while online friends can be added at virtually no cost. This paper contains some diagrams which is non-understandable. It does not have any impressive future plan as well.

Long et al. [16] worked on Exploring TrajectoryDriven Local Geographic Topics in Foursquare. It focuses on exploring the local geographic topics through check-ins In Pittsburgh area in Foursquare. It uses the Latent Dirichlet Allocation (LDA) model to discover the local geographic topics from the check-ins. It also compares the local geographic topics on weekdays with those at weekends. It contains well-understandable and relative diagrams which is helpful in understanding the motives of the paper. The weak point of the paper is that it has no well- suited future plan, and do not show any progress of the future development.

Chow et al. [17] worked on Towards Location based social Networking services. This paper has strong abstract that present an aggregate system called Geo Social DB. It provides three location-based social networking services namely; location-based news feed, locationbased news ranking, and location-based recommendation. The purpose of Geo Social DB is to implement the aforementioned services as query operators inside a database engine to optimize the query processing performance. Within the Geo Social DB framework, it also discusses research challenges, and directions toward the realization of scalable and practical query processing for locationbased social networking services. Some of the titles names are not well-suited. Some of its diagrams are lucid. There is no future enhancement progress.

On the basis of the above discussion the following Table 1 is drawn which shows the existing social net work frameworks, their comparison, major categories and supported applications. At the end of the table a proposed framework is suggested that supports all the major categories and applications.

\section{Preliminary}

This Section of the paper presents the key structure of GMBSN system that elaborates how users from the worldwide web can access the system from any location on the globe. In order to access the GMBSN system the user will first has to visit GMBSN. After that they will be followed by a page presenting highlighted cities on the map. The user will then select any city highlighted on the map. After selecting the city, users have to select the category of their desired interest/need. In the category page user will be able to share and get useful information

Table 1. Comparison of the proposed framework with the existing frameworks.

\begin{tabular}{|c|c|c|c|c|c|c|c|c|c|c|c|c|}
\hline \multirow[t]{2}{*}{$\begin{array}{l}\text { Reference } \\
\text { No. }\end{array}$} & \multicolumn{6}{|c|}{$\begin{array}{l}\text { Social Networks Based on } \\
\text { Inside/Outside Country }\end{array}$} & \multicolumn{6}{|c|}{ Major Categories/Supported Applications } \\
\hline & $\begin{array}{l}\text { Inside } \\
\text { Country }\end{array}$ & $\begin{array}{l}\text { Outside } \\
\text { Country }\end{array}$ & Both & Tourism & Shopping & Restaurants & Living Areas & Business & $\begin{array}{c}\text { Hospitals/ } \\
\text { Health centers }\end{array}$ & Sports & $\begin{array}{l}\text { Educational } \\
\text { Institutes }\end{array}$ & $\begin{array}{c}\text { Special } \\
\text { Events/ } \\
\text { Others }\end{array}$ \\
\hline$[18]$ & & & $\checkmark$ & & & & & $\checkmark$ & & & & $\checkmark$ \\
\hline$[20]$ & & & $\checkmark$ & & & & & & & & & $\checkmark$ \\
\hline [21] & $\checkmark$ & & & & & & & & & $\checkmark$ & & $\checkmark$ \\
\hline$[22]$ & & & $\checkmark$ & & $\checkmark$ & $\checkmark$ & & & & & & $\checkmark$ \\
\hline [23] & & & $\checkmark$ & & & $\checkmark$ & & & & & & $\checkmark$ \\
\hline$[24]$ & & & $\checkmark$ & $\checkmark$ & $\checkmark$ & $\checkmark$ & & & & & & \\
\hline$[26]$ & & & $\checkmark$ & $\checkmark$ & & & & & & & & $\checkmark$ \\
\hline $\begin{array}{r}\text { Proposed } \\
\text { Framework } \\
\end{array}$ & $\checkmark$ & $\checkmark$ & $\checkmark$ & $\checkmark$ & $\checkmark$ & $\checkmark$ & $\checkmark$ & $\checkmark$ & $\checkmark$ & $\checkmark$ & $\checkmark$ & $\checkmark$ \\
\hline
\end{tabular}


about the specified city in the form of posts. The phenomena are described in the following Figure 3.

\section{GMBSN Architecture}

GMBSN, functions for the provision of multifarious essential information for the ease of worldwide clients on the globe. The users of social network should visit in tyro/novice to GMBSN which cited in Figure 4. Shortly after, going through the required process, GMBSN clients shall choose a particular city from the map. The map can be accessed through Google map API as described earlier. Soon after selecting a city, users will be redirected to the city page, where they can have another option for selecting category from the categories index, depending on their interest as directed in Figures 3 and 4. When the category selection process gets over, client will be re-enacted to Category page: there will be innumerable information about the likened city of a country related to category. Users will then be enabled to watch out all the posts; but they are ought to authenticate the claim before rating, commenting or sharing the visual posts, the process for authentication is shown in Figure 4. Insofar as, if the clients attempt to make any particular action they shall seek to login their Facebook account, method have shown in Figure 4. The purpose of authentication is to provide an access to the users for the performance of certain actions inside GMBSN. For query, users will be directed to "Facebook login's page" where they shall be required to login their Facebook accounts, if a user already is a member of GMBSN then he/she shall make haste to redirect it, otherwise it will show a pop up to the user for granting permission to access GMBSN, already shown in Figure 4. After the authentication process user can eagerly evaluate/devaluate the post.

\section{System Functions}

GMBSN provide some basic functions for their users which includes the following basic functions.

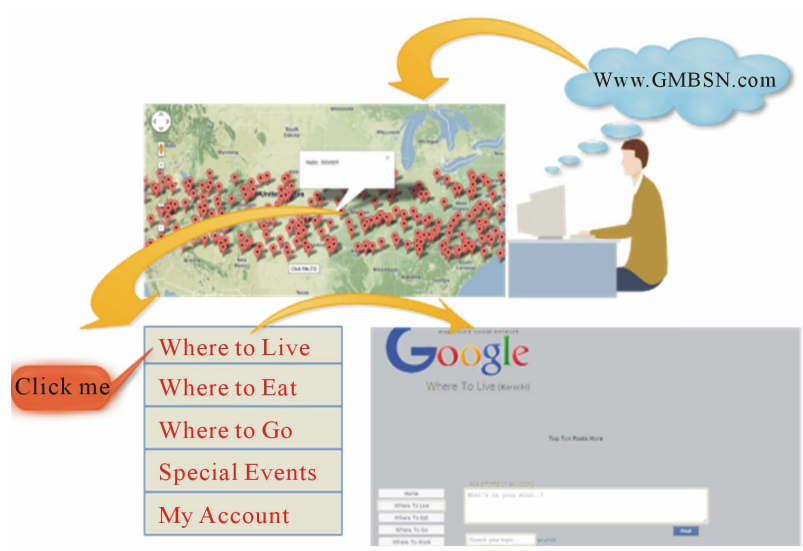

Figure 3. GMBSN user interface.

\subsection{Google Map}

GMBSN system utilizes Google map which discussed in the aforementioned sections. It is friendlier/fun-loving activity for users and easy to interact with, hence it will be handy enough for clients to watch the map and explore any region being highlighted on the map and will be redirected to that region on the globe.

\subsection{Categories}

GMBSN provide different categories in overall system which includes home, where to live, where to eat, where to go and my account etc $\cdots$. These parts can be explained and divided into three sub-categories on the basis of their functions e.g.

1) Home: This is the category when user click on this category he/she will be redirected to the main page and it is the crucial one, since if the user might be anywhere, and on any page, they can directly switch-over to the main page by clicking this category.

2) Middle Categories: These includes categories e.g. where to live, to eat and to go etc $\cdots$ These categories provide easy access for users to go to any category of their interest or according to their need of information about the concern region. Users can choose any category from the index and can be easily entered to the related category.

User can switch-over from one category to another simultaneously.

3) My Account: This category includes the overall information about the user who is using this network. The information includes the users Facebook profile name, picture and any other information, and the most important information such as user's posts, comments, and likes etc $\cdots$. This category also contains the friend list of those users who are added with the current user. It also consists of find new friends which suggest friends to current user to add them. These users are suggested on the basis of best ratings or comments on the current user's post. Users can add these suggested users by just clicking on add as friend button and can easily remove these friends by just clicking on remove button.

\section{Conclusion}

This paper presents a Google map-based social network. This recommended system can facilitate people's travel not only near their living areas but also to a city that is new to them (even if they have not been to any place). Users all around the globe can get any sort of useful information about any region all around the world by just visiting and get involving in this network. Users of this network have no security problems because this network authenticate every user through their Facebook account, since Facebook is the most usable social net work across 


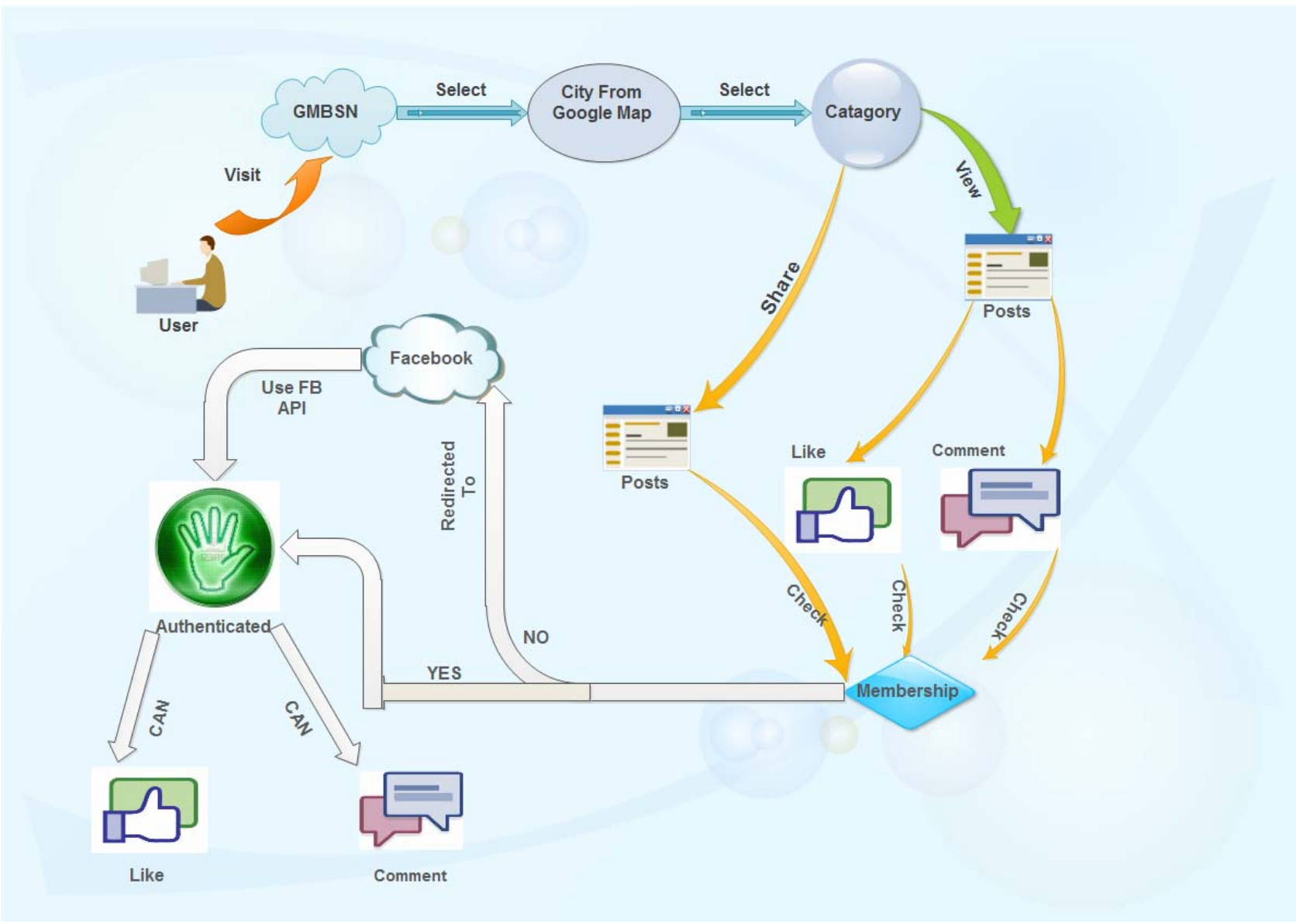

Figure 4. GMBSN architecture.

the globe and have currently expanded its vendors in order to provide best protection over one billion users who use this network [27]. Currently we have implemented this system for Pakistan and in future we are looking to spread and implement this system in all over the world.

\section{REFERENCES}

[1] N. Li and G. Chen, "Analysis of a Location-Based Social Network," International Conference on Computational Science and Engineering, Vancouver, 29-31 August 2009, pp. 263-270. doi:10.1109/CSE.2009.98

[2] M. Ye, P. Yin and W.-C. Lee, "Location Recommendation for Location-Based Social Networks," Proceedings of the 18th SIGSPATIAL International Conference on Advances in Geographic Information Systems, (c), (2010, pp. 458. doi:10.1145/1869790.1869861

[3] I. Workshop, "We Will Set One Best Paper award According to the Review Organizers General Chair: Program Chair: Program Committee," The 4th International Workshop on Location-Based Social Networks (LBSN 2012), Pittsburgh, 8 September 2012.

[4] C. Fu, Y. Wang, Y. Xu and Q. Li, "The Logistics Network System Based on the Google Maps API," 2010 Interna- tional Conference on Logistics Systems and Intelligent Management (ICLSIM), Harbin, 9-10 January 2010, pp. 1486-1489. doi:10.1109/ICLSIM.2010.5461215

[5] H. Li and L. Zhijian, "The Study and Implementation of Mobile GPS Navigation System Based on Google Maps," 2010 International Conference on Computer and Information Application, Tianjin, 3-5 December 2010, pp. 8790. doi:10.1109/ICCIA.2010.6141544

[6] S. Saha, N. Agarwal, P. Dhanuka and S. Nandi, "Google Map Based User Interface for Network Resource Planning in Post Disaster Management," Proceedings of the 3rd ACM Symposium on Computing for Development, Bangalore, 11-12 January 2013, Article No. 22. doi: $10.1145 / 2442882.2442910$

[7] http://www.sdbay.sdsu.edu/maps/images/DisMap_archite cture.gif

[8] http://developers.facebook.com/docs/reference/php/faceb ook-api/

[9] http://uswaretech.com/blog/wp-content/uploads/2009/02/f acebook.jpg

[10] J. J.-C .Ying, W.-C. Lee, M. Ye, C.-Y. Chen and V. S. Tseng, "User Association Analysis of Locales on Location Based Social Networks," Proceedings of the 3rd ACM SIGSPATIAL International Workshop on LocationBased Social Networks, Chicago, 1 November 2011, pp. 
69-76. doi: $10.1145 / 2063212.2063214$

[11] J. Bao, Y. Zheng and M. F. Mokbel, "Location-Based and Preference-Aware Recommendation Using Sparse GeoSocial Networking Data," Proceedings of the 20th International Conference on Advances in Geographic Information Systems SIGSPATIAL, Redondo Beach, 6-9 November 2012, pp. 199-208. doi: $10.1145 / 2424321.2424348$

[12] D. Yamamoto, I. Takumi and H. Matsuo, "Location-Based Social Network Services Employing Student Cards for University," Proceedings of the 2009 International Workshop on Location Based Social Networks, Seattle, 3 November 2009, pp. 21-24. doi:10.1145/1629890.1629895

[13] S. C. Tan, "Location Aware Applications for Smart Cities with Google Maps and GIS Tools," 2006.

[14] S. Scellato and C. Mascolo, "Measuring User Activity on an Online Location-Based Social Network," 2011 IEEE Conference on Computer Communications Workshops (INFOCOM WKSHPS), Shanghai, 10-15 April 2011, pp. 918-923. doi:10.1109/INFCOMW.2011.5928943

[15] X. Long, L. Jin and J. Joshi, "Exploring Trajectory-
Driven Local Geographic Topics in Foursquare," Proceedings of the 2012 ACM Conference on Ubiquitous Computing, Pittsburgh, 5-8 September 2012, pp. 927-934. doi: $10.1145 / 2370216.2370423$

[16] http://www.facebook.com

[17] C. Chow, "Towards Location-Based Social Networking Services Categories and Subject Descriptors," 2010.

[18] http://georillas.com/

[19] http://eu1.badoo.com/

[20] http://www.shakabanga.com/

[21] http://www.dehood.com/http://www.foursquare.com

[22] http://www.citysourced.com/about

[23] http://www.foodspotting.com

[24] http://www.foursquare.com

[25] http://en.friendticker.com/

[26] http://www.locr.commap

[27] http://www.facebook.com/security 